\title{
A Cross-Shape Coil Structure for Use in Wireless Power Applications
}

\author{
Seyit Ahmet Sis * (1) and Emre Orta \\ Electrical and Electronics Engineering, Balikesir University, Balikesir 10145, Turkey; emreorta3@gmail.com \\ * Correspondence: seyit.sis@balikesir.edu.tr; Tel.: +90-507-018-8285
}

Received: 6 April 2018; Accepted: 24 April 2018; Published: 28 April 2018

\begin{abstract}
This paper presents a novel coil structure employing two parallel-connected subunit rectangular coils. The structure is based on the fact that the rectangular coils are less sensitive to misalignment along its longer side. Therefore, two identical subunit rectangular coils are vertically-oriented to one another and connected in parallel to form a cross-shape coil. The cross-shape coils have the advantage of better misalignment tolerance as compared to circular and square coils with similar footprints at the cost of an increased wire usage. Electromagnetic simulations and experiments on various small-sized coils are performed to verify the advantages of the proposed coil structure. Based on the simulation and measurement results, cross-shape coils exhibit not only better tolerance to misalignment, but also smaller self-inductance values resulting in larger coupling coefficients as compared to square and circular coils. A large cross-shape coil pair consisting of $100 \times 70 \mathrm{~cm}$ subunit rectangles are fabricated with copper tubes and utilized in a frequency-tuned wireless power transfer system. When coils are separated by $17 \mathrm{~cm}$, a near constant efficiency of more than $89 \%$ up to $15 \mathrm{~cm}$ misalignment along $x$ - or $y$-directions and $13 \mathrm{~cm}$ along diagonal direction is obtained in the frequency-tuned system.
\end{abstract}

Keywords: wireless power transfer; cross-shape coil; circular coil; square coil; misalignment; mutual inductance

\section{Introduction}

Magnetic resonance-based wireless power transfer systems (MRWPTs) offer larger transfer distances as compared to inductive power transfer systems (IPTs). This makes MRWPTs suitable for applications ranging from electric vehicle chargers and automated guided vehicles (AGVs) to mobile devices such as laptops and mobile phones [1-4]. In all these applications, the device to be charged may be misaligned during power transfer and such a misalignment needs to be considered in the system design, because it reduces the coupling between coils and ultimately deteriorates the power transfer efficiency.

Various system-level solutions have been proposed for compensating the output power reductions due to coupling variations [5-15]. Tunable impedance-matching circuits, or so-called impedance-tuned systems, may be utilized to match the source and load resistances to varying input and output impedances seen toward the transmit and receive coils, respectively [5-9]. Impedance-tuned systems utilize tunable lumped components and a control unit. Frequency-tuned systems are also proposed by researchers to deliver a constant power to the load against varying coupling between the coils [11-15]. One can maintain an almost-constant output power at strongly coupled regime if the radio frequency (RF) source frequency is tuned to either one of the two resonance frequencies, which are known as even- and odd-mode frequencies ( $f_{\text {even }}$ and $\left.f_{\text {odd }}\right)$.

In addition to the aforementioned system-related solutions, significant efforts have been made to optimize the coils for increasing tolerance to misalignment between coils [16-23]. Bipolar coil 
structures such as flux-pipe coils consist of two parallel connected helical-shape coils which are wound around a common ferrite core extending along the $x$-direction. A magnetic flux pipe is formed between these parallel connected coils, which creates a longer fundamental flux path along both $x$ - and $z$-(height) directions. Therefore, high coupling level and improved misalignment tolerance is obtained in flux-pipe coils. A major disadvantage of the flux pipe coils is that they exhibit double-sided magnetic flux distribution. Therefore, when a metallic shield is used at the back side of coil, quality factor $(Q)$ of the coil reduces significantly. A single-sided double-D (DD) shape bipolar structure, which also forms a magnetic flux pipe along the $x$-direction toward which ferrites are extending, is proposed for improving coupling level and misalignment tolerance [17]. In DD coils, wires are wound on top of the ferrite cores, forming two D-shape planar coils which are electrically connected in parallel. Each D-shape coil is placed back-to-back such that number of turns doubles at the middle of the DD coil. This is equivalent to magnetically connecting each D-shape coil in series with each other, creating larger coupling coefficients. DD coils exhibit good misalignment tolerance toward only $y$-direction, which is parallel to the wires at the middle of the DD coil [17]. When a misalignment is applied along the $x$-direction (along the flux pipe length), DD coils exhibit large sensitivity to misalignment. To increase tolerance to misalignment along the $x$-direction, a quadrature coil is added to the DD coil and this new combined coil is referred to as a DDQ coil [17]. The DDQ coil exhibits very good tolerance to lateral misalignment along both $x$ - and $y$-directions; however, the DD and $Q$ coils have no electrical connection and need separate circuitry. For example, if the DDQ coil is utilized at the secondary side, DD and $Q$ coils are independently tuned and the signals coming from these coils are rectified separately [17]. Therefore, complexity and cost of wireless power transfer systems with DDQ coils are higher as compared to systems with only DD coils. Depending on the application, lateral misalignment may be more important in one direction than in others; in this case, one can use DD coils and place them according to the misalignment requirement $[18,19]$. In a recently reported work, a bipolar DD coil and a unipolar rectangular shape coil is integrated into a single coil structure which exhibits large coupling coefficients with smaller sizes and improvements in tolerance to misalignment [21]. However, as in DDQ coils, the integrated bipolar and unipolar coils are also connected to separate circuits at both primary and secondary sides. Therefore, inverter and compensation components at the primary side, as well as compensation components and rectifiers at the secondary side, are doubled as compared to traditional wireless power transfer systems. Three-dimensional loops, utilizing a three-loop structure, are also presented and they show a nearly angle-insensitive efficiency [22]. A drawback in three-dimensional loops would be the difficulty associated with mounting the loops on devices. A quad-D-quadrature (QDQ) coil structure, consisting of four adjacent circular coils and a rectangular coil, has been proposed for reducing the sensitivity to the misalignment of coupled coils [23].

Besides these novel coil structures, optimizing compensation capacitors [24] or utilizing multiple component compensation such as a series-parallel-series (SPS) compensation topology [25] can provide more than $30 \%$ improvement in tolerance to the misalignment. Finally, nonlinear resonators, represented by duffing equations, have recently been proposed to compensate for coupling variations and exhibit substantial improvement to misalignment and range variations [26]. A near-constant $75 \%$ efficiency has been reported up to $18 \mathrm{~cm}$ coil separations [26]. These efforts on coil geometry and compensation networks, which may be combined with system level solutions discussed in the previous paragraph, offer a considerable potential for realizing a coupling-variation tolerant wireless power transfer system.

\section{Contribution}

In this paper, we first study lateral misalignment performance of typical square, circular, and rectangular coils. Findings are discussed by briefly addressing the differences between misalignment tolerance performances of these three common coil structures. Subsequently, a new coil structure—cross-shape coil—is proposed by utilizing the findings from this study. Cross-shape coils 
have the advantage of exhibiting better lateral misalignment tolerance as compared to square and circular coils. These advantages are verified through both simulations and measurement of fabricated small-sized coils using a vector network analyzer. Measurement results unveil another advantage, that the cross-shape coils have larger mutual inductance to self-inductance ratios, which in turn results in larger magnetic coupling coefficients as compared to square and circular coils. Considering these advantages, a large-sized cross-shape coil pair is finally fabricated using copper tubes and utilized in a frequency-tuned wireless power transfer system.

\section{Misalignment Analysis of Square, Circular, Rectangular, and Cross-Shape Coils}

\subsection{Square, Circular and Rectangular Coils}

Mutual inductance of single-turn circular, square, and rectangular coil pairs is studied using an electromagnetic High Frequency Structural Simulator (HFSS). The diameter and side lengths of circular and square coils are chosen as $30 \mathrm{~cm}$ in simulations. Rectangular coils with various aspect ratios are simulated and the longer side of all rectangular coils are fixed to $30 \mathrm{~cm}$. Figure 1 shows typical HFSS model drawings for simulated single-turn coupled coil pairs along with the direction vectors. In HFSS simulations, the conductors for all coil types are modeled as a solid copper wire with a diameter of $1 \mathrm{~mm}$. Coils are placed at the center of a cubic air box with a side length of $200 \mathrm{~cm}$ and excited via $50 \mathrm{ohm}$ lumped ports. Misalignment is applied along $x-, y-$, and diagonal directions, and for each misalignment, 2-port S-parameters are simulated for the coupled coil pairs from $100 \mathrm{kHz}$ to $2000 \mathrm{kHz}$ with $100 \mathrm{kHz}$ steps. The mutual inductance $(M)$ is extracted by utilizing a 2-port equivalent circuit model of magnetically coupled coils as shown in Figure 1a. This model can further be manipulated to a T-network as seen in Figure 1b [27]. Analyzing the model in Figure $2 b$ reveals the following expression for $M$ [28]:

$$
M=\frac{Z_{21}}{j \times\left(2 \pi f_{0}\right)}
$$

and

$$
Z_{21}=\frac{2 S_{21}}{\left(1-S_{11}\right)\left(1-S_{22}\right)-S_{21} S_{12}}
$$

where $S_{11}, S_{12}, S_{21}$ and $S_{22}$ are the simulated 2-port S-parameters for the coupled coils.

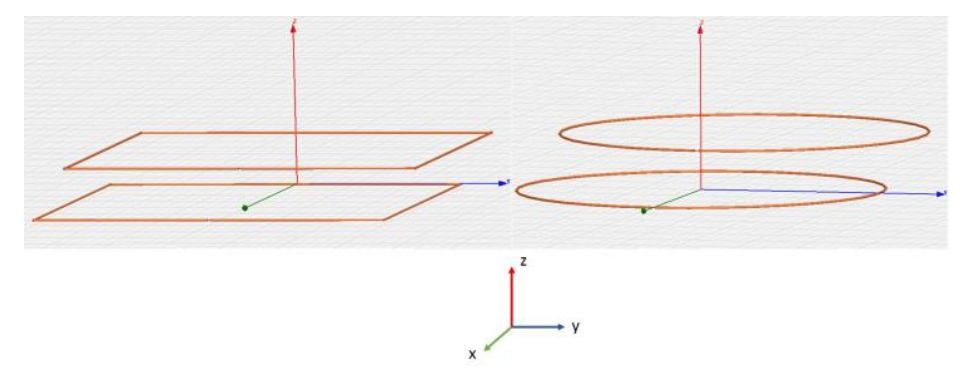

Figure 1. HFSS model drawings for coupled rectangular and circular coil pairs along with $x$-, $y$ - and $z$-direction vectors.

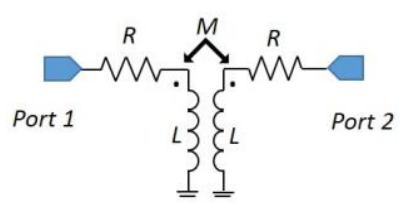

(a)

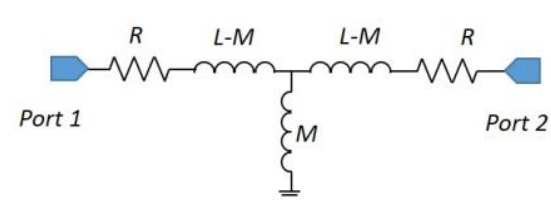

(b)

Figure 2. (a) A typical and (b) T-equivalent circuit models for magnetically coupled coils as two-port networks. Both circuits exhibit the same electrical response. 
In HFSS simulations, the shorter sides of all rectangular coils are in parallel with $x$-direction. Figure $3 \mathrm{a}, \mathrm{b}$ show normalized mutual inductances as a function of misalignments along $x$ - and $y$-directions, respectively, for single-turn square, circular, and rectangular coils. In these simulations, the separation between the coils is $5 \mathrm{~cm}$. The nominal mutual inductance values, for perfectly aligned situations $\left(M_{\max }\right)$ and $5 \mathrm{~cm}$ coil separation, are given in the graphs for each coupled coil pairs. As seen in Figure $3 \mathrm{a}, \mathrm{b}$, square coils exhibit better tolerance than the circular coils to both $x$ - and $y$-direction misalignments. Since shorter sides of rectangular coils are parallel with $x$-direction (see Figure 1), any misalignment along $x$-direction predominantly increase the separation between longer sides of rectangular coil pairs. Therefore, rectangular coils are more sensitive to misalignment along the $x$-direction as compared to square and circular coils as seen in Figure 3a. Sensitivity of rectangular coils to $x$-direction misalignment gets higher as the aspect ratio reduces. When misalignment is applied along the longer sides ( $y$-direction), rectangular coils exhibit higher tolerance to misalignment as compared to circular and square coils (Figure $3 b$ ), because misalignment along the $y$-direction predominantly increases the separation between the shorter sides of the rectangular coil pairs. These misalignment characteristics of rectangular coils can be exploited to form a new coil structure, details of which are presented in following section.

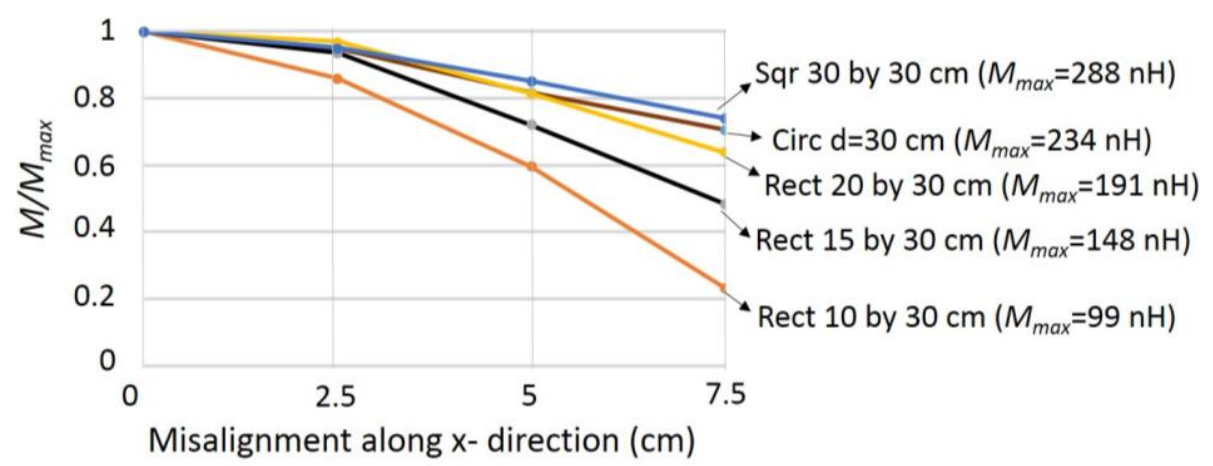

(a)

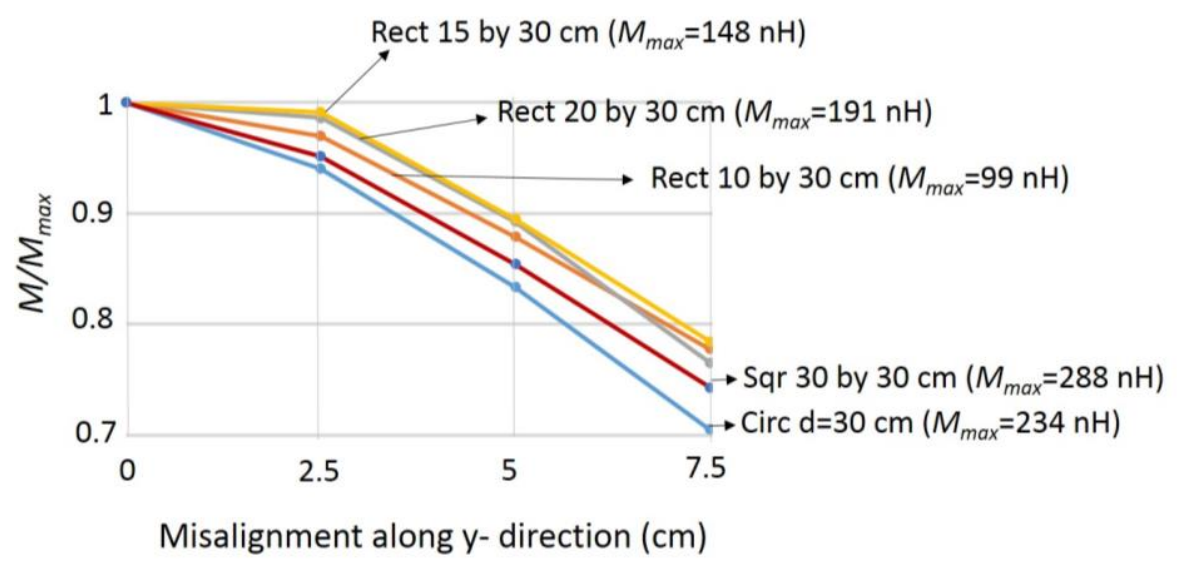

(b)

Figure 3. Normalized mutual inductance $\left(M / M_{\max }\right)$ for single turn square, circular, and rectangular coils as a function misalignment along (a) $x$-direction and (b) $y$-direction. Separation between coils is $5 \mathrm{~cm}$.

\subsection{Cross-Shape Coils}

Cross-shape coils consist of two rectangular subunit coils which are vertically aligned to one another with the same geometric midpoints as shown in Figure 4. The subunit rectangular coils are electrically connected in parallel and the inputs are connected in such a way that the current on each 
rectangular coil flows through the same direction. Therefore, the magnetic field created by each coil becomes constructive at the middle of cross-shape structure. As discussed in the previous section, rectangular coils exhibit better tolerance to misalignment along their longer sides as compared to square and circular coils (Figure $3 \mathrm{~b}$ ). Therefore, cross-shape coils are expected to show improved tolerance to misalignment along any direction on horizontal plane. Initial verification is performed via HFSS simulations on single-turn cross-shape coils for various lateral misalignments. Figure 5 shows an HFSS model drawing of a simulated cross-shape coil pair.

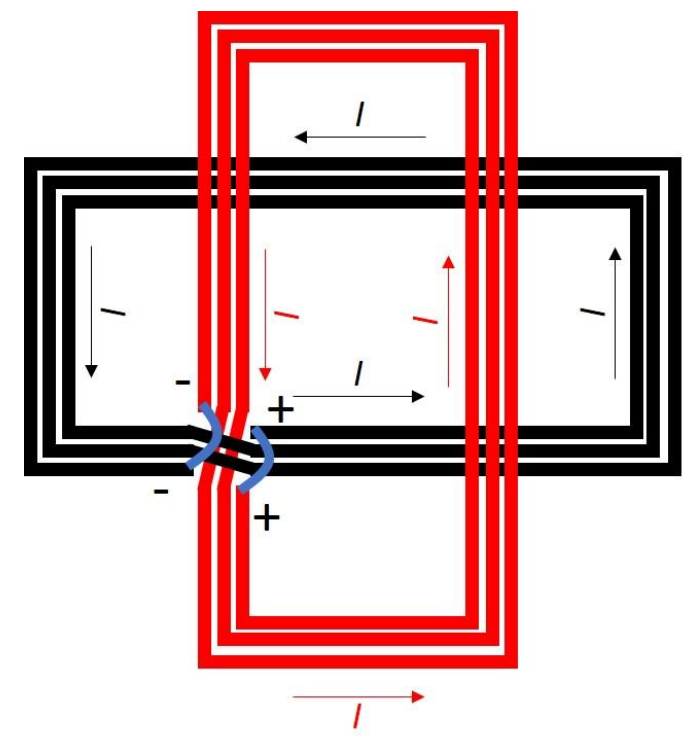

Figure 4. Proposed cross-shape coil structure.

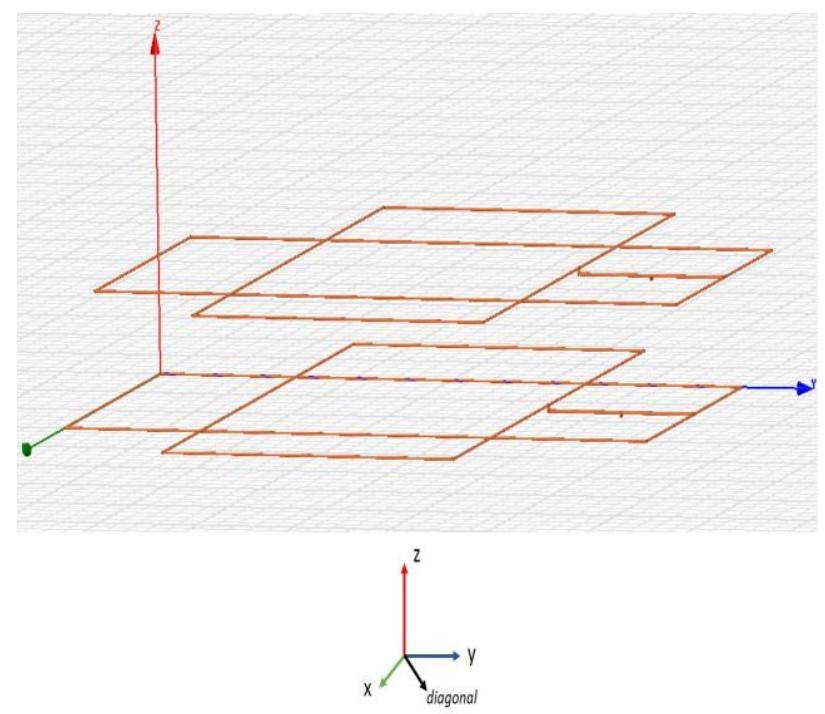

Figure 5. An HFSS model drawing for coupled cross-shape coil pairs and $x-, y-, z-$, and diagonal direction vectors.

Lateral misalignments are applied along both $x$ - and $y$-directions and the diagonal direction (see Figure 5) during simulations. The $M$ values of coupled cross-shape coils for each misaligned situation are extracted from simulated 2-port $S$-parameters using (1). Normalized mutual inductances of the two cross-shape coils-a large one with $20 \times 30 \mathrm{~cm}$ (Cross 20 by $30 \mathrm{~cm}$ ) and a small one with $17 \times 30 \mathrm{~cm}$ (Cross 17 by $30 \mathrm{~cm}$ ) subunit rectangular coils-are compared to those of square and circular 
coils as a function of misalignment along $x$ - (or $y$-) and diagonal directions as shown in Figure 6 . Nominal $M$ values for perfectly aligned coils $\left(M_{\max }\right)$ with $5 \mathrm{~cm}$ separation are also given on the same graphs. Since coils are symmetric around the $x$ and $y$ axes, misalignments along $x$ - and $y$-directions have the same effect on coupling between the coils.

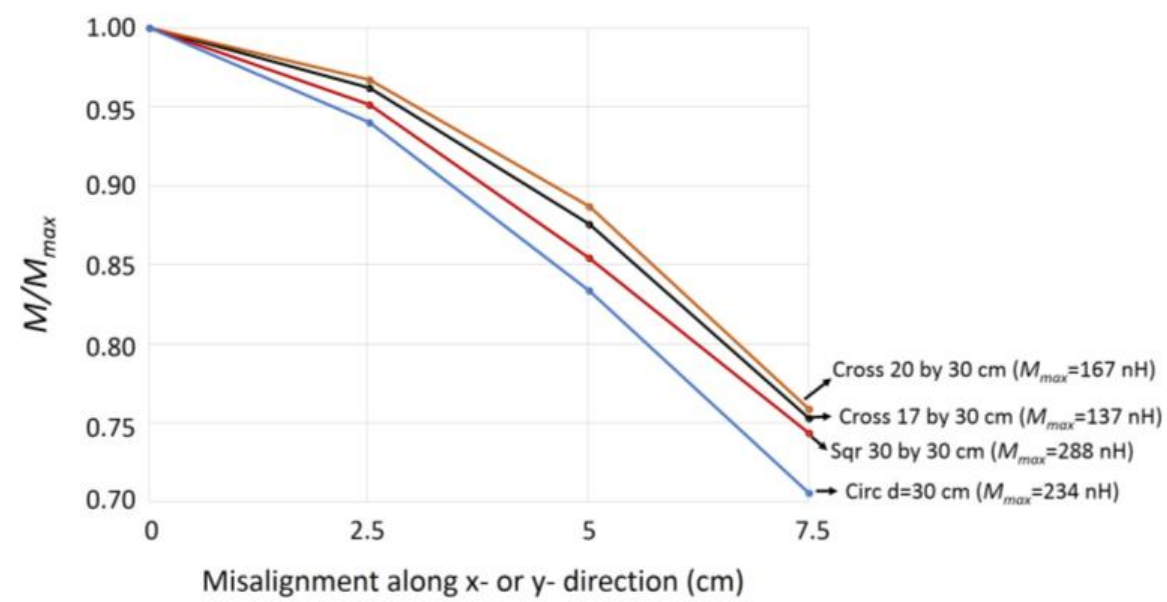

(a)

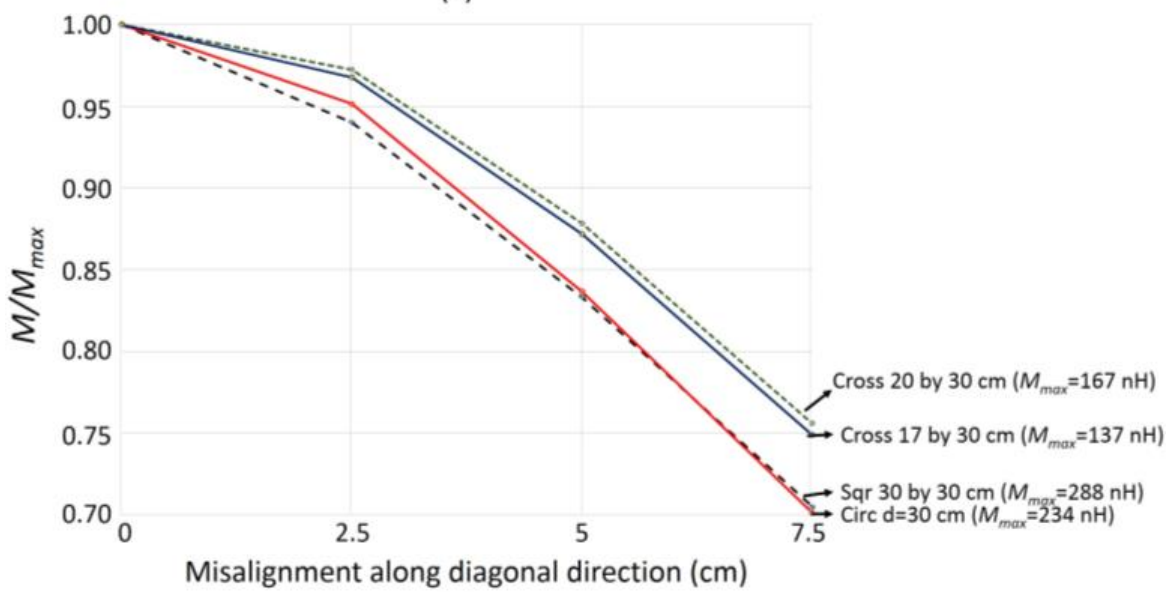

(b)

Figure 6. Normalized mutual inductance $\left(M / M_{\max }\right)$ for single-turn cross-shape, square and circular coils as a function misalignment along (a) $x$ - (or $y$-) direction and (b) diagonal direction. These mutual inductance values are extracted from simulated 2-port S-parameters. Separation between coils is $5 \mathrm{~cm}$.

As seen in Figure 6a, cross-shape coils exhibit better tolerance to misalignment along the $x$-direction as compared to both circular and square coils. For example, when $5 \mathrm{~cm}$ misalignment is applied, small and large cross-shape coils exhibit $2.7 \%$ and $4.7 \%$ better tolerance, respectively, than the square coils. The same small and large cross-shape coils exhibit $6.4 \%$ and $8.2 \%$ better tolerance, respectively, to $5 \mathrm{~cm}$ misalignment along $x$-direction as compared to circular coils. When misalignments are applied along the diagonal direction, improvement of tolerance in cross-shape coils is even more apparent, as seen in Figure $6 \mathrm{~b}$. At $7.5 \mathrm{~cm}$ misalignment along the diagonal direction, cross-shape coils exhibit more than $7 \%$ higher tolerance than the square and circular coils. During simulations, it was observed that misalignment tolerance of cross-shape coils is no better than square coils when the short sides of subunit rectangular coils are smaller than $15 \mathrm{~cm}$. Therefore, the aspect ratio of subunit rectangular coils in cross-shape coils need to be smaller than two. A major drawback of cross-shape coils is that they exhibit smaller coupling levels as compared to square and circular coils as seen in $M_{\max }$ values in Figure 6. Therefore, one needs to increase the number of turns $(N)$ to obtain adequate mutual inductance values. Increasing the wire usage initially seems disadvantageous but, as will be discussed 
in following paragraph, parallel connection of subunit rectangular coils makes the self-inductance and parasitic loss resistance of cross-shape coils even lower than those of square and circular coils.

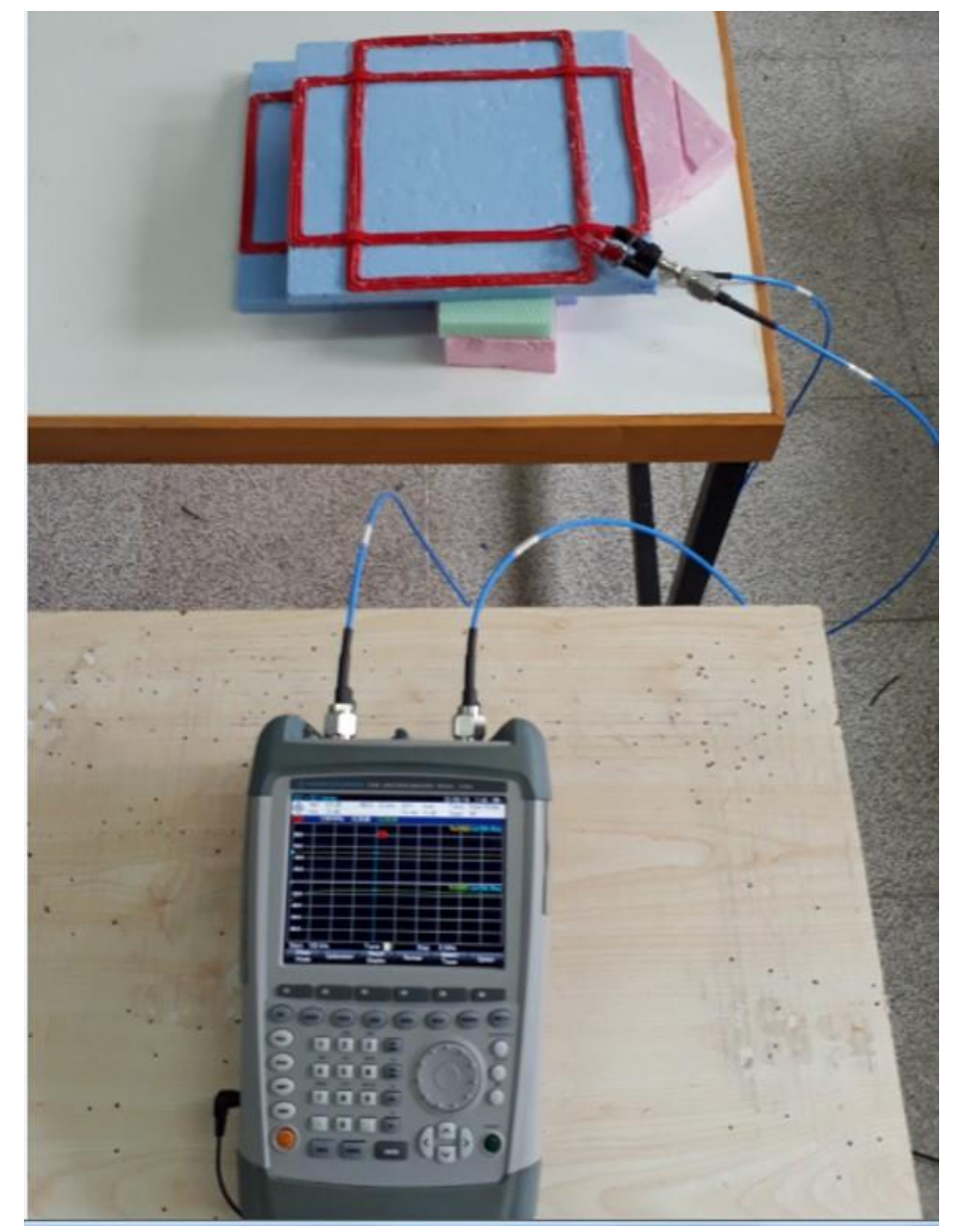

Figure 7. An S-parameter measurement setup for fabricated multi-turn coils.

Multi-turn square, circular, and cross-shape coils are fabricated for analyzing the effect of the excessive wire usage on cross-shape coils. Three-turn $(N=3)$ square and circular coils with side length and diameter of $30 \mathrm{~cm}$, respectively, and cross-shape coils with four-turn, $20 \times 30 \mathrm{~cm}$ rectangular subunit coils are fabricated on Styrofoam. In all coils, solid copper wires with $0.9 \mathrm{~mm}$ radius are utilized. These multi-turn coils are measured using a using Rohde and Schwarz FSH8 Spectrum + Network analyzer ( Rohde \& Schwarz, Munich, Germany) from $100 \mathrm{kHz}$ to $5 \mathrm{MHz}$ as shown in Figure 7. Misalignments are applied along $x-, y$-, and diagonal directions during S-parameter measurements.

Mutual inductance values are extracted from measured 2-port S-parameters using Equation (1). Figure $8 \mathrm{a}, \mathrm{b}$ show normalized mutual inductance as a function of misalignments along $x$ - (or $y$ - due to symmetry) and diagonal directions, respectively. Cross-shape coils exhibit substantial improvement in terms of tolerance to misalignment along both $x$ - and diagonal directions, verifying simulation results of single-turn coils shown in Figure 6. The maximum mutual inductance value, when each coil pair is perfectly aligned with $5 \mathrm{~cm}$ separation, are represented by $M_{\max }$ and shown for each coil type on the graphs in Figure 8. One can see that the mutual inductance values of four-turn cross-shape coils are larger than that of three-turn square and circular coils. In all coil types, a typical $N^{2}$ relationship can be observed between mutual inductance and number of turns by comparing the simulation results of single-turn coils in Figure 6 with measurement results of multi-turn coils in Figure 8. 


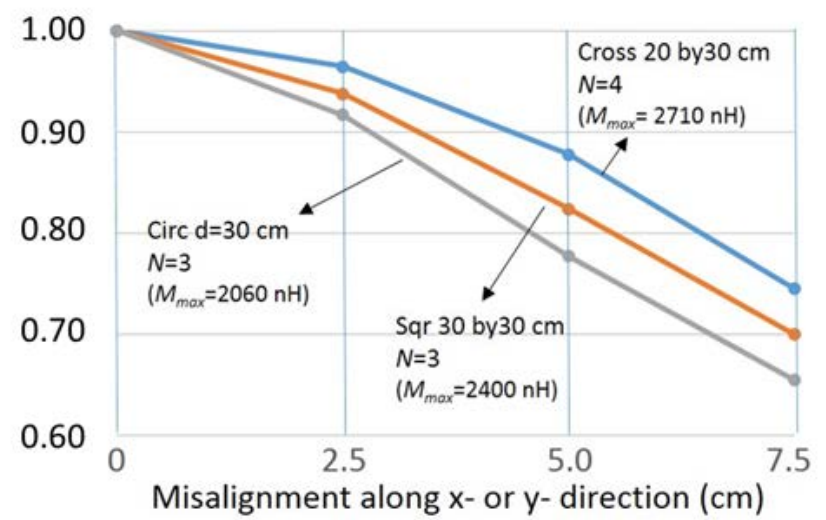

(a)

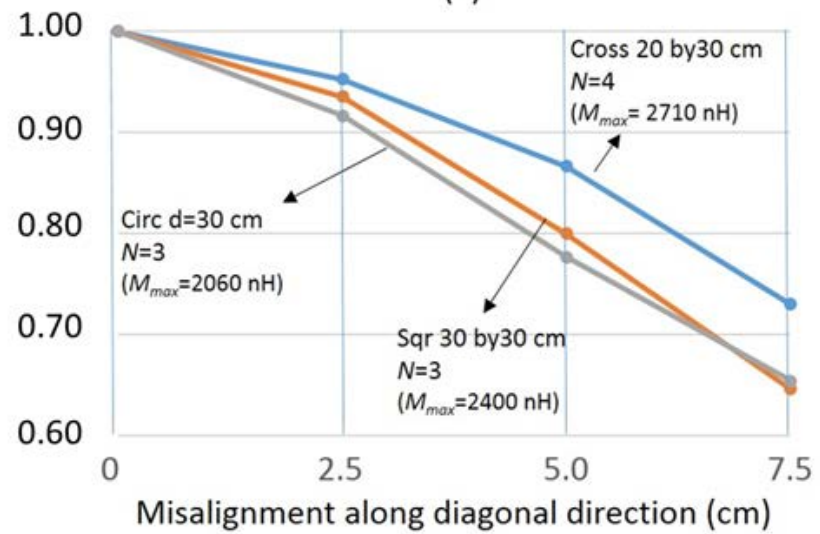

(b)

Figure 8. Normalized mutual inductance $\left(M / M_{\max }\right)$ for multi-turn cross-shape, square and circular coils as a function misalignment along (a) $x$ - (or $y$-) direction and (b) diagonal direction. These mutual inductance values are extracted from measured 2-port S-parameters. Separation between coils are $5 \mathrm{~cm}$.

The self-inductance and parasitic loss-resistance for each coil is extracted from measured 2-port $S$-parameters of each uncoupled coil in a series configuration as shown in Figure 9a. Losses are represented as a series-connected loss resistance $\left(R_{\text {loss }}\right)$ to self-inductance $(L)$ as shown in Figure $9 \mathrm{~b}$ and can be extracted from the measured $S_{11}$, which is the input reflection coefficient when the second port is loaded with system impedance, using Equations (2) and (3) as follows:

$$
\begin{gathered}
R_{\text {loss }}=\operatorname{Re}\left[\frac{2 Z_{0} \cdot S_{11}}{1-S_{11}}\right] \\
L=\frac{\operatorname{Im}\left[\frac{2 Z_{0} \cdot S_{11}}{1-S_{11}}\right]}{2 \pi f}
\end{gathered}
$$

where $Z_{0}$ is the $50 \Omega$ system impedance of the network analyzer. The extracted $R_{\text {loss }}$ values increase with frequency due to frequency-dependent $\mathrm{AC}$ and radiation resistances. To make a comparison, average value of $R_{\text {loss }}$ resistances within a measured frequency range along with extracted $L$ values for each coil are given in Table 1 . The $M$ value for perfectly aligned identical coils with $5 \mathrm{~cm}$ separation, $M / L$ ratio, area and wire usage for each coil are also given in Table 1 to make a comprehensive comparison between all coil types. As seen in Table 1, cross-shape coils have larger wire usage for achieving an equal to or more mutual inductance values than circular and square coils; however, the loss resistance of cross-shape coil is slightly smaller despite this larger wire usage. Total wire used in four-turn $20 \times 30 \mathrm{~cm}$ cross-shape coil is $800 \mathrm{~cm}$, which is larger than $283 \mathrm{~cm}$ and $360 \mathrm{~cm}$ wire usage of three-turn circular and square coils, respectively. However, average loss resistance of cross-shape coil 
is $0.75 \Omega$, which is still slightly smaller than the $0.8 \Omega$ and $0.9 \Omega$ average loss resistances of circular and square coils, respectively (Table 1 ). Since subunit rectangular coils are electrically parallel, cross-shape coils exhibit larger $M / L$ values as compared to square and circular coils as seen in Table 1 . Therefore, cross-shape coils exhibit larger coupling coefficient values, which in turn results in higher power levels delivered to the load. For example, for a typical resonant inductive wireless power transfer system with identical transmit and receive coils, the output power $\left(P_{\text {out }}\right)$ is directly proportional to $(M / L)$ and can be expressed as follows [17]:

$$
P_{\text {out }}=\omega I_{1}{ }^{2} \frac{M^{2}}{L} Q_{r}=V_{1} I_{1} k^{2} Q_{r}
$$

where $\omega, I_{1}, V_{1}, Q_{r}$, and $k$ are radial frequency, current flowing through transmit coil, voltage across the transmit coil, operating quality factor of receiving side, and magnetic coupling coefficient, respectively. A small $L$ value is important also in high-power systems for limiting the voltage swings below the maximum voltage ratings at the transmitter side [29]. In the following section, a frequency-tuned wireless power transfer system utilizing a large size cross-shape coil pair is presented.

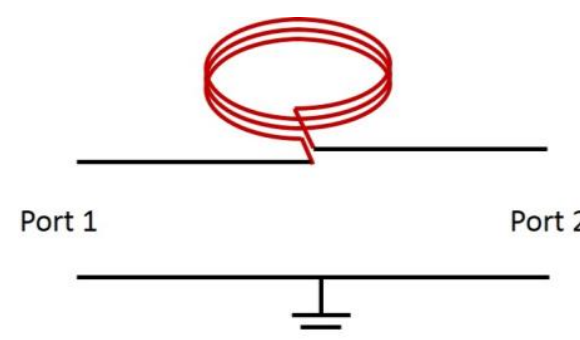

(a)

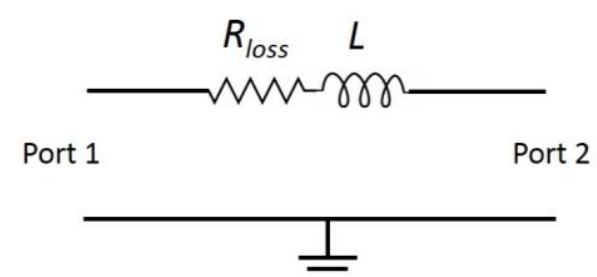

(b)

Figure 9. (a) 2-port S-parameter measurement scheme of each coil in a series configuration, (b) electrical model of the coil in the same configuration.

Table 1. The extracted electrical parameters, area, and wire usage for each fabricated coil. $M$ values are for when coils are perfectly aligned with $5 \mathrm{~cm}$ separation.

\begin{tabular}{c|c|c|c|c|c|c}
\hline $\begin{array}{c}\text { Parameters } \\
\text { Coil Type }\end{array}$ & $R_{\text {loss }}$ & $\boldsymbol{L}$ & $\boldsymbol{M}$ & $\boldsymbol{M} / \mathbf{L}$ & Area & Wire Usage \\
\hline $\begin{array}{c}\text { Circular Coil } \\
(d=30 \mathrm{~cm}, N=3) \\
\text { Square Coil }\end{array}$ & $0.8 \Omega$ & $6670 \mathrm{nH}$ & $2060 \mathrm{nH}$ & 0.308 & $706 \mathrm{~cm}^{2}$ & $283 \mathrm{~cm}$ \\
$\begin{array}{c}(l=30 \mathrm{~cm}, N=3) \\
\text { Cross-Shape Coil } \\
(20 \times 30 \mathrm{~cm}, N=4)\end{array}$ & $0.9 \Omega$ & $8000 \mathrm{nH}$ & $2400 \mathrm{nH}$ & 0.300 & $900 \mathrm{~cm}^{2}$ & $360 \mathrm{~cm}$ \\
\hline
\end{tabular}

\section{A Frequency-Tuned Wireless Power Transfer System with Cross-Shape Coils}

In this section, five-turn $(N=5)$ cross-shape coils with $100 \times 70 \mathrm{~cm}$ rectangular subunits are fabricated using copper tubes with inner radius and copper thickness of $635 \mu \mathrm{m}$ and $70 \mu \mathrm{m}$, respectively. First, the self-inductance of fabricated cross-shape coils are extracted as $34.3 \mu \mathrm{H}$ using Equation (3). The fabricated coils are then utilized in a frequency-tuned system, which has been proposed in the literature as a system-level solution for compensating the output power reductions due to the coupling variations [11-15]. The block diagram of the frequency-tuned system is shown in Figure 10. Frequency of operation is chosen as $550 \mathrm{kHz}$; therefore, the value of series connected compensation capacitors is approximately $2.4 \mathrm{nF}$. An RF signal generator with source impedance of $50 \Omega$ is connected to the input port of a $20 \mathrm{~dB}$ bidirectional coupler. The bidirectional coupler exhibits $50 \Omega$ at all its four ports; therefore, the source impedance $\left(R_{S}\right)$ in the frequency-tuned system is $50 \Omega$ as well. The power is 
delivered to a load resistance $\left(R_{L}\right)$ of a $25 \Omega$ resistor as shown in Figure 10. The picture of tested frequency-tuned system with fabricated cross-shape coils is shown in Figure 11.

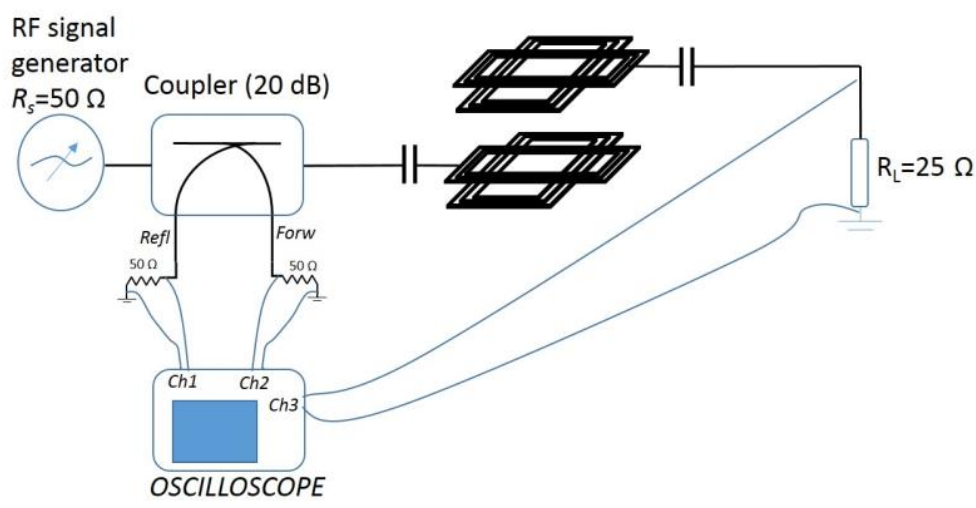

Figure 10. Block diagram of frequency-tuned system.

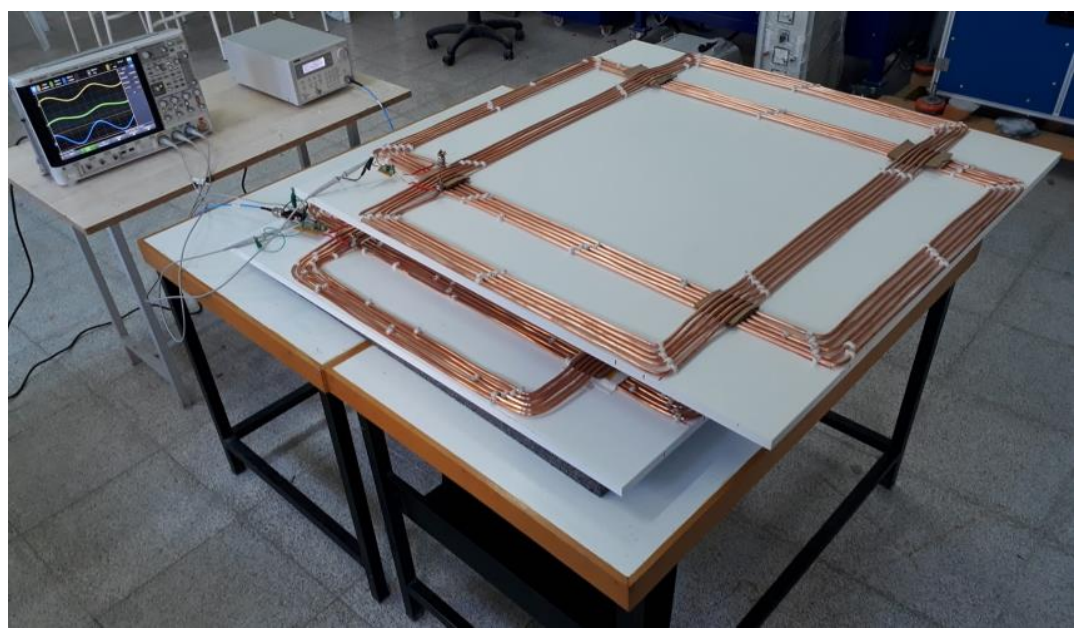

Figure 11. Picture of tested frequency tuned-system utilizing fabricated cross-shape coils.

The output power of the RF signal generator is set to $7 \mathrm{dBm}$. Misalignment is applied between coils and, at each misalignment, the frequency of the RF signal generator is varied manually until the power at the reflect port is minimum. This guarantees the frequency of highest power transfer efficiency by just controlling the system at transmitter side. At this frequency, the power delivered to load $\left(P_{L}\right)$ is calculated by using the measured voltage across $R_{L}$. Subsequently, peak power transfer efficiency is calculated as the ratio of power delivered to the load to the power available from the source $\left(\eta=P_{L} / P_{A V S}\right)$. Figure 12a shows measured peak efficiencies as a function of misalignment along the $x$ - (or $y$-) direction. Figure 12b shows these resonance frequencies as function of misalignment along the $x$ - (or $y$-) direction. The coils are separated by $17 \mathrm{~cm}$ along the $z$-direction when misalignments are applied. As seen in Figure 12a, a near-constant efficiency of more than $89 \%$ is obtained up to $15 \mathrm{~cm}$ misalignment along the $x$-direction. Beyond $15 \mathrm{~cm}$, efficiency drops significantly with increasing lateral misalignment. The tracked resonance frequency, at which peak efficiency is obtained (Figure 12b), reduces from $586 \mathrm{kHz}$ to approximately $550 \mathrm{kHz}$ as misalignment increases from 0 to $15 \mathrm{~cm}$. Beyond $15 \mathrm{~cm}$, resonance frequency stays almost constant at $550 \mathrm{kHz}$ as lateral misalignment increases. One can conclude that that coils are in a strongly coupled regime for up to a critical-coupling misalignment of $15 \mathrm{~cm}$. It was observed that the peak efficiency is obtained slightly above the resonance frequency of isolated coils $\left(f_{0}=550 \mathrm{kHz}\right)$ due to the frequency splitting phenomenon at strongly coupled regimes. Figure 13 shows similar $\eta_{\max }$ and $f_{r}$ graphs for diagonal misalignments. 
Critical coupling misalignments, up to which a near constant efficiency of more than $89 \%$ is obtained, is approximately $13 \mathrm{~cm}$.

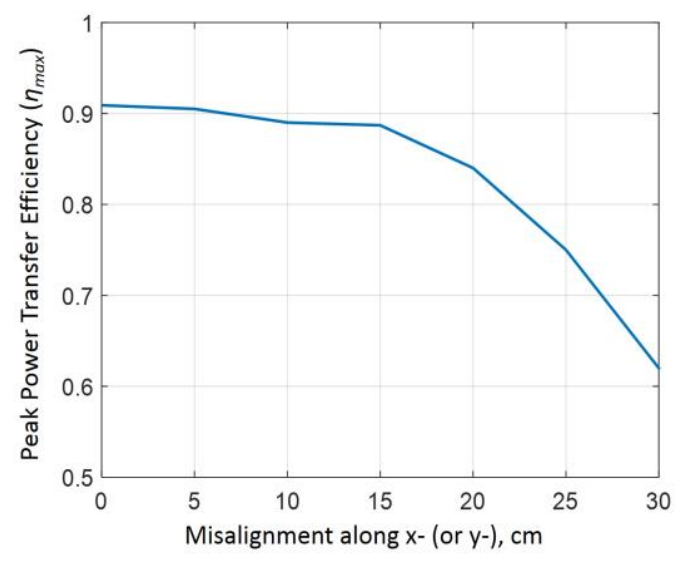

(a)

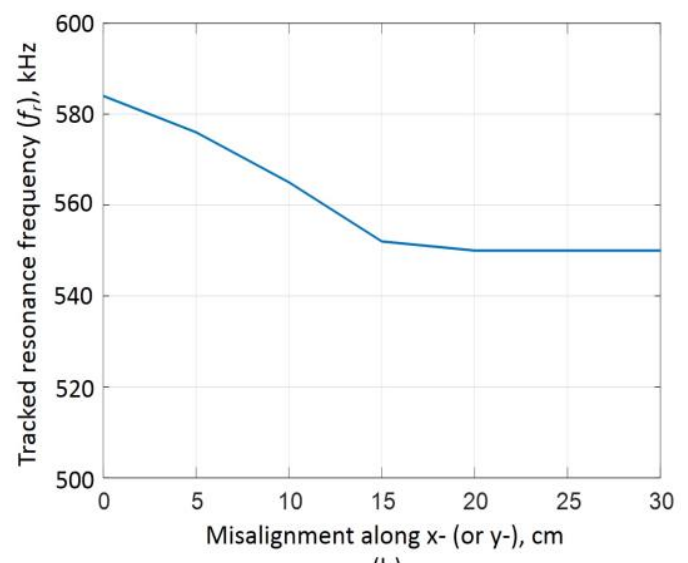

(b)

Figure 12. (a) Peak power transfer efficiency and (b) tracked resonance frequency as function of lateral misalignment along $x$-direction.

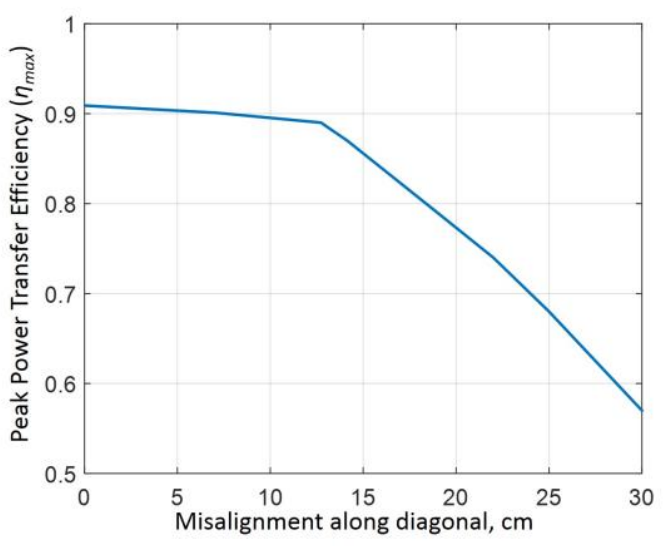

(a)

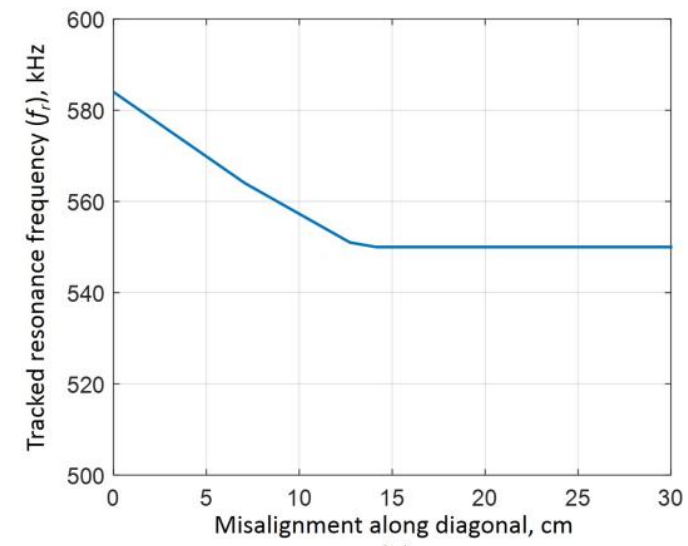

(b)

Figure 13. (a) Peak power transfer efficiency and (b) tracked resonance frequency as function of lateral misalignment along diagonal-direction.

\section{Misalignment Tolerance Comparison with Bipolar Coils}

The fabricated large-sized cross-shape coil pairs, utilized in a frequency-tuned system above, are characterized in terms of mutual inductance. For this, 2-port S-parameters of magnetically coupled coil pairs are measured using Rohde and Schwarz FSH8 Spectrum + Network analyzer from $100 \mathrm{kHz}$ to $5 \mathrm{MHz}$. When measuring 2-port S-parameters, misalignments up to $25 \%(25 \mathrm{~cm})$ of a side length of the cross-shape coil $(100 \mathrm{~cm})$ are applied at each $5 \%$ misalignment steps. $M$ and $L$ values are extracted from measured 2-port S-parameters using Equations (1) and (3), respectively.

Misalignment tolerance performance of proposed cross-shape coil is compared to that of a $77 \times 41 \mathrm{~cm}$ DD coil reported in [17]. The mutual inductance $(M)$ for DD coils as function of misalignment is not directly given, but uncompensated power $\left(P_{s u}\right)$ is plotted as a function of misalignments along $x$ - and $y$-directions in [17]. One can extract mutual inductance from $P_{s u}$ values using Equation (5) as follows:

$$
M=\sqrt{\frac{P_{s u} L}{\omega I_{1}^{2}}}
$$


where $L / \omega I_{1}$ can be taken as a constant that can easily be calculated from given $M$ and $P_{s u}$ values in [17] for the perfectly aligned coils. Then one can solve $M$ values for given $P_{s u}$ as a function of misalignment along the $x$ - and $y$-directions. Figure 14 shows normalized mutual inductance $\left(M / M_{\max }\right)$ as a function of percentage misalignments along $x$ - and $y$-directions relative to the longer side of DD $(77 \mathrm{~cm})$ and cross-shape coils $(100 \mathrm{~cm})$. As seen in Figure 14, cross-shape coils exhibit the same tolerance to misalignments along both $x$ - and $y$-directions, which are nearly same with DD coils' tolerance to the $y$-direction. The tolerance of DD coils gets significantly worse when misalignment is applied along the $x$-direction.

The DDQ coils reported in [17] exhibit much better tolerance to misalignment along the $x$-direction as compared to both DD and cross-shape coils; however, in DDQ coils, separate circuits are needed for DD and $Q$ coils, making the wireless power system more complex. Flux pipe coils are another type of bipolar coil with large mutual inductance and lateral misalignment tolerance; however, due to their non-planar windings around I-shape ferrites, they are double-sided coils and may exhibit high losses if shielding is placed on the back side of the coils. Here the reported cross-shape coils are planar and suitable for forming a single-sided magnetic flux distribution if ferrites are placed underneath the windings.

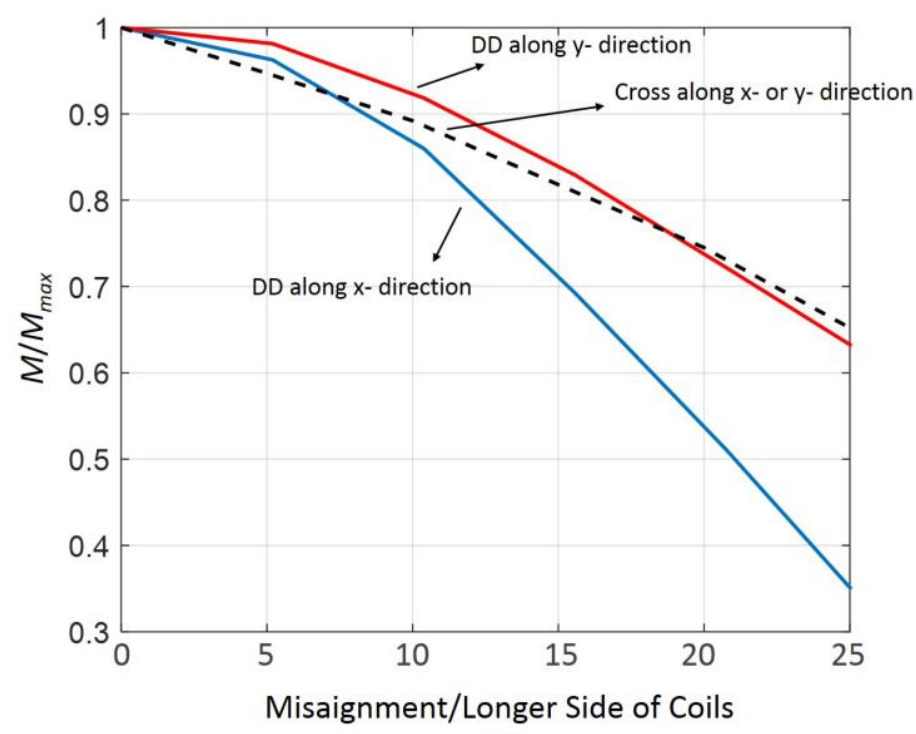

(\%)

Figure 14. Normalized mutual inductance $\left(M / M_{\max }\right)$ for cross-shape, and DD coils as a function misalignment along $x$ - and $y$-directions.

\section{Conclusions}

In this paper, a novel cross-shape coil structure, employing two parallel connected subunit rectangular coils, are presented for use in wireless power applications. Simulations and experimental verifications show that the proposed coil exhibits better lateral misalignment tolerance at the cost of larger wire usage as compared to commonly used circular and square coils. Larger wire usage, on the other hand, seems to be a major disadvantage; however, this larger wire usage does not increase the loss resistance and self-inductance of cross-shape coils due to parallel connection of the subunit rectangular coils. In fact, measurement results show that cross-shape coils exhibit larger $M / L$ ratios as compared to circular and square coils with similar lateral size and mutual inductance values. Here the proposed coil structure is not meant to be an alternative to various other recently reported novel coil structures such as DD and QDD coils. Nevertheless, misalignment performance of cross-shape coils is discussed also by comparing it with that of DD coils in Section 3. That comparison shows that cross-shape coils exhibit the same high tolerance along both orthogonal directions in the horizontal 
plane while DD coils exhibit high tolerance to misalignment only along one orthogonal direction. The mutual inductances of DD coils are expected to be larger as they exhibit longer fundamental flux paths as compared to unipolar coils. Here, the mutual inductances are not directly compared because size of coils are different, and no ferrites have been utilized in cross-shape coils. Cross-shape coils can be manipulated to fit any desired application without ruining its misalignment tolerance advantages. For example, ferrite materials can be placed under copper windings to create a single-sided magnetic field distribution for use in electric vehicle charging systems.

\section{Patents}

A patent application was made to the Turkish Patent and Trademark Office on cross-shape coil structure on 6 April 2018.

Author Contributions: Seyit Ahmet Sis proposed the coil structure. Both Seyit Ahmet Sis and Emre Orta (graduate student) performed simulations. Seyit Ahmet Sis fabricated and measured the coils with help of undergrad students. Seyit Ahmet Sis wrote the paper and Emre Orta checked for grammar errors.

Acknowledgments: Authors thank to undergraduate students Umut Uzun and Artur Klychov for helping in fabricating the large size coils.

Conflicts of Interest: The authors declare no conflict of interest. The founding sponsors had no role in the design of the study; in the collection, analyses, or interpretation of data; in the writing of the manuscript, and in the decision to publish the results.

\section{References}

1. Li, S.; Mi, C.C. Wireless Power Transfer for Electric Vehicle Applications. IEEE J. Emerg. Sel. Top. Power Electron. 2015, 3, 4-17.

2. Raabe, S.; Covic, G.A.; Boys, J.T.; Pennalligen, C.; Shekar, P. Practical considerations in the design of multiphase pick-ups for contactless power transfer systems. In Proceedings of the 2009 35th Annual Conference of IEEE Industrial Electronics, Porto, Portugal, 3-5 November 2009; pp. 753-758.

3. Zaheer, A.; Covic, G.A.; Kacprzak, D. A Bipolar Pad in a 10-kHz 300-W Distributed IPT System for AGV Applications. IEEE Trans. Ind. Electron. 2014, 61, 3288-3301. [CrossRef]

4. Taylor, J.A.; Low, Z.N.; Casanova, J.; Lin, J. A wireless power station for laptop computers. In Proceedings of the 2010 IEEE Radio and Wireless Symposium (RWS), New Orleans, LA, USA, 10-14 January 2010; pp. 625-628.

5. Lim, Y.; Tang, H.; Lim, S.; Park, J. An Adaptive Impedance-Matching Network Based on a Novel Capacitor Matrix for Wireless Power Transfer. IEEE Trans. Power Electron. 2014, 29, 4403-4413. [CrossRef]

6. Beh, T.C.; Kato, M.; Imura, T.; Oh, S.; Hori, Y. Automated Impedance Matching System for Robust Wireless Power Transfer via Magnetic Resonance Coupling. IEEE Trans. Ind. Electron. 2013, 60, 3689-3698. [CrossRef]

7. Waters, B.H.; Sample, A.P.; Smith, J.R. Adaptive impedance matching for magnetically coupled resonators. In Proceedings of the PIERS, Progress in Electromagnetics Research Symposium Proceedings, Moscow, Russia, 19-23 August 2012; pp. 694-701.

8. Heebl, J.D.; Thomas, E.M.; Penno, R.P.; Grbic, A. Comprehensive Analysis and Measurement of Frequency-Tuned and Impedance-Tuned Wireless Non-Radiative Power-Transfer Systems. IEEE Antennas Propag. Mag. 2014, 56, 131-148. [CrossRef]

9. Duong, T.P.; Lee, J.-W. A Dynamically Adaptable Impedance-Matching System for Midrange Wireless Power Transfer with Misalignment. Energies 2015, 8, 7593-7617. [CrossRef]

10. Hwang, K.; Cho, J.; Kim, D.; Park, J.; Kwon, J.H.; Kwak, S.I.; Park, H.H.; Ahn, S. An Autonomous Coil Alignment System for the Dynamic Wireless Charging of Electric Vehicles to Minimize Lateral Misalignment. Energies 2017, 10, 315. [CrossRef]

11. Gao, Y.; Zhou, C.; Zhou, J.; Huang, X.; Yu, D. Automatic Frequency Tuning with Power-Level Tracking System for Wireless Charging of Electric Vehicles. In Proceedings of the 2016 IEEE Vehicle Power and Propulsion Conference (VPPC), Hangzhou, China, 17-20 October 2016; pp. 1-5.

12. Kar, D.P.; Nayak, P.P.; Bhuyan, S.; Panda, S.K. Automatic frequency tuning wireless charging system for enhancement of efficiency. Electron. Lett. 2014, 50, 1868-1870. [CrossRef] 
13. Sis, S.A.; Bicakci, S. A resonance frequency tracker and source frequency tuner for inductively coupled wireless power transfer systems. In Proceedings of the 2016 46th European Microwave Conference (EuMC), London, UK, 4-6 October 2016; pp. 751-754.

14. Luo, Y.; Yang, Y.; Chen, S.; Wen, X. A frequency-tracking and impedance-matching combined system for robust wireless power transfer. Int. J. Antennas Propag. 2017. [CrossRef]

15. Seo, D.W.; Lee, J.H. Frequency-Tuning Method Using the Reflection Coefficient in a Wireless Power Transfer System. IEEE Microw. Wirel. Compon. Lett. 2017, 27, 959-961. [CrossRef]

16. Budhia, M.; Covic, G.; Boys, J. A new IPT magnetic coupler for electric vehicle charging systems. In Proceedings of the IECON 2010-36th Annual Conference on IEEE Industrial Electronics Society, Glendale, AZ, USA, 7-10 November 2010; pp. 2487-2492.

17. Budhia, M.; Boys, J.T.; Covic, G.A.; Huang, C.Y. Development of a Single-Sided Flux Magnetic Coupler for Electric Vehicle IPT Charging Systems. IEEE Trans. Ind. Electron. 2013, 60, 318-328. [CrossRef]

18. Nguyen, T.D.; Li, S.; Li, W.; Mi, C.C. Feasibility study on bipolar pads for efficient wireless power chargers. In Proceedings of the 2014 IEEE Applied Power Electronics Conference and Exposition-APEC 2014, Fort Worth, TX, USA, 16-20 March 2014; pp. 1676-1682.

19. Deng, J.; Li, W.; Nguyen, T.D.; Li, S.; Mi, C.C. Compact and Efficient Bipolar Coupler for Wireless Power Chargers: Design and Analysis. IEEE Trans. Power Electron. 2015, 30, 6130-6140. [CrossRef]

20. Wei, Q.; Guo, W.; Sun, X.; Wang, G.; Zhao, X.; Li, F.; Li, Y. A New Type of IPT System with Large Lateral Tolerance and its Circuit Analysis. In Proceedings of the 2012 International Conference on Connected Vehicles and Expo (ICCVE), Beijing, China, 12-16 December 2012; pp. 311-315.

21. Li, Y.; Lin, T.; Mai, R.; Huang, L.; He, Z. Compact Double-Sided Decoupled Coils-Based WPT Systems for High-Power Applications: Analysis, Design, and Experimental Verification. IEEE Trans. Transp. Electrification 2018, 4, 64-75. [CrossRef]

22. Jonah, O.; Georgakopoulos, S.V.; Tentzeris, M.M. Orientation insensitive power transfer by magnetic resonance for mobile devices. In Proceedings of the 2013 IEEE Wireless Power Transfer (WPT), Perugia, Italy, 15-16 May 2013; pp. 5-8.

23. Kalwar, K.A.; Mekhilef, S.; Seyedmahmoudian, M.; Horan, B. Coil design for high misalignment tolerant inductive power transfer system for EV charging. Energies 2016, 9, 937. [CrossRef]

24. Zhu, Q.; Guo, Y.; Wang, L.; Liao, C.; Li, F. Improving the Misalignment Tolerance of Wireless Charging System by Optimizing the Compensate Capacitor. IEEE Trans. Ind. Electron. 2015, 62, 4832-4836. [CrossRef]

25. Villa, J.L.; Sallan, J.; Osorio, J.F.S.; Llombart, A. High-Misalignment Tolerant Compensation Topology for ICPT Systems. IEEE Trans. Ind. Electron. 2012, 59, 945-951. [CrossRef]

26. Wang, X.; Abdelatty, O.; Mortazawi, A. A novel coupling factor independent highly efficient resonant based wireless power transfer. In Proceedings of the 2017 47th European Microwave Conference (EuMC), Nuremberg, Germany, 10-12 October 2017; pp. 200-203.

27. Sis, S.A. Ferroelectric-on-Silicon Switchable Bulk Acoustic Wave Resonators and Filters for RF Applications. Ph.D. Dissertation, University of Michigan, Ann Arbor, MI, USA, June 2014.

28. Pozar, D.M. Microwave Engineering; John Wiley \& Sons: Hoboken, NJ, USA, 2012; p. 192.

29. Covic, G.A.; Kissin, M.L.G.; Kacprzak, D.; Clausen, N.; Hao, H. A bipolar primary pad topology for EV stationary charging and highway power by inductive coupling. In Proceedings of the 2011 IEEE Energy Conversion Congress and Exposition, Phoenix, AZ, USA, 17-22 September 2011; pp. 1832-1838.

(C) 2018 by the authors. Licensee MDPI, Basel, Switzerland. This article is an open access article distributed under the terms and conditions of the Creative Commons Attribution (CC BY) license (http://creativecommons.org/licenses/by/4.0/). 